\title{
FISIOTERAPIA E SAÚDE COLETIVA: UMA ANÁLISE CRITTICA DA PRODUÇÃO CIENTÍFICA NO BRASIL
}

\author{
PHYSICAL THERAPY AND COLLECTIVE HEALTH: A CRITICAL ANALYSIS \\ OF THE SCIENTIFIC PRODUCTION IN BRAZIL
}

\author{
Carolina Veras Pessoa da Silva \\ Graduada em Fisioterapia pela Universidade Federal \\ da Bahia (UFBA)

\section{Milena Maria Cordeiro de Almeida} \\ Doutoranda em Saúde Coletiva do Instituto de \\ Saúde Coletiva, UFBA
}

\author{
Contato \\ Carolina Veras Pessoa da Silva \\ Rua Araújo Pinho, n 503, bloco A, apt. 802. \\ Bairro Canela, Salvador/BA \\ CEP: 40110150 \\ E-mail: carolinapessoa15@gmail.com
}

\section{RESUMO}

Introdução: A sistematização crítica dos saberes e práticas da atuação da Fisioterapia na Saúde Coletiva (SC) pode contribuir para uma transformação da atuação profissional, extrapolando as práticas tradicionais de reabilitação e ampliando o interesse dos profissionais de Fisioterapia por esse campo. Desenvolvimento: Revisão de literatura com o objetivo de realizar uma análise crítica da produção científica no Brasil sobre a Fisioterapia e a Saúde Coletiva. A busca foi realizada nas bases de dados Bireme, SciELO e Lilacs utilizando os descritores "Fisioterapia" and "Saúde Coletiva", selecionando-se 14 publicações. Foram encontradas um maior número de publicações em 2011, com maior predomínio na região Sudeste e atuação na Atenção Básica. Os estudos foram discutidos nos seguintes eixos: 1) Formação para Saúde Coletiva ou Saúde Pública; 2)
Integralidade nas práticas da Fisioterapia e Saúde Coletiva; 3) Interdisciplinaridade nas práticas da Fisioterapia e Saúde Coletiva. Considerações finais: A produção científica da Fisioterapia na SC cresceu nos últimos anos, mas de forma incipiente comparada a outras áreas de atuação. Os estudos estão concentrados nas práticas assistenciais, sem extrapolar para uma reflexão crítica sobre os pressupostos do campo. Percebe-se uma dificuldade na realização de práticas integrais e interdisciplinares, devido à desarticulação da formação profissional com a prática em saúde, reafirmando a necessidade de aproximação da Fisioterapia a SC, para que os profissionais estejam capacitados para contribuir na construção de política públicas, no levantamento de dados epidemiológicos de saúde, na gestão de serviços de saúde e na articulação da saúde com as ciências sociais.

Palavras-chave: Fisioterapia; Saúde Coletiva; Sistema Único de Saúde. 


\section{ABSTRACT}

Introduction: A critical systematization of knowledge and practices in the performance of Physical therapy in Public Health (SC) may contribute to a transformation of professional action, extrapolating the traditional rehabilitation practices and broadening the interests of physical therapy professionals in this field. Development: Literature review in order to carry out a critical analysis of the scientific production in Brazil on the Physical therapy and Public Health. The search was conducted in the Bireme, Lilacs and SciELO databases using the descriptors "Physical Therapy" and "Public Health", having selected 14 publications. A greater number of publications were found in 2011, with highest prevalence in the Southeast and performance in Primary Care. The studies were discussed in the following areas: 1) Training for Collective or Public He- alth; 2) Integrality in Physical therapy and Public Health practices; 3 ) Interdisciplinarity in Physiotherapy and Public Health practices of Physiotherapy and Public Health. Final Thoughts: The scientific production of Physical therapy in SC has grown in recent years, but in an incipient way compared to other areas. The studies are focused on care practices, without extrapolating to a critical reflection on the assumptions of the field. We note a difficulty in achieving comprehensive and interdisciplinary practices due to the disarticulation of professional training with practice in health, reaffirming the need for approximation of Physical therapy to SC, so that professionals are able to contribute to construction of public policies, in the survey of epidemiological health data, in health care management and in the integration of health with the social sciences.

Keywords: Physical Therapy Specialty; Public Health; Unified Health System.

\section{INTRODUÇÃO}

$\mathrm{O}$ avanço da Fisioterapia no Brasil tem sido marcado pela expansão do objeto de intervenção e do campo de atuação. Ao longo dos anos, amplificou-se sua atuação reabilitadora centrada no restabelecimento da capacidade física de indivíduos após a ocorrência de doenças e obteve reconhecimento social e profissional na área da saúde. O desenvolvimento da profissão aconteceu predominantemente com foco na atenção individual e reabilitadora, apresentando uma baixa mobilização dos profissionais para uma atuação coletiva e orientada pela Promoção à Saúde ${ }^{1}$.

As transformações políticas, ideológicas e organizativas que aconteceram no setor saúde foram acompanhadas tardiamente pela profissão. A Fisioterapia, enquanto categoria organizada, não participou de forma expressiva do processo de Reforma Sanitária nos anos 1970, ao contrário das outras profissões de saúde, iniciando essas discussões nas entidades representativas apenas a partir dos anos 2000 , ainda assim, marcada por grandes interesses mercadológicos ${ }^{1}$.

Podemos destacar a publicação tardia, mas não menos importante, da Resolução Coffito 363/2009 que reconhece a Fisioterapia em Saúde Coletiva como especialidade profissional do fisioterapeuta, estabelecendo que ela pode ser exercida por meio de ações básicas de saúde, na fisioterapia do trabalho, em programas institucionais e na vigilância sanitária².

A atuação do fisioterapeuta na Saúde Coletiva (SC) está em processo de construção, com um avanço no que se refere à regulamentação desta no Sistema Único de Saúde (SUS), no entanto ainda se faz necessário um debate crítico a respeito das práticas profissionais e dos marcos teóricos e conceituais que embasam essa atuação. A incorporação das ações de prevenção e promoção da saúde e o exercício de uma nova lógica de organização dos modelos assistenciais são alguns efeitos dessas mudanças na Fisioterapia ${ }^{3}$. A perspectiva da SC na Fisioterapia surge como alternativa a esses novos desafios e necessidades para reorientação do foco de atenção e da prática profissional ${ }^{3}$.

A promoção da sistematização e reflexão crítica sobre os saberes e práticas na SC, em um contexto de poucas publicações científicas, poderia contribuir para uma reflexão e transformação da atuação do fisioterapeuta, extrapolando as práticas tradicionais de reabilitação e ampliando o interesse e engajamento dos profissionais de Fisioterapia por esse campo de prática. Esse estudo, portanto, teve por objetivo realizar o levantamento e análise crítica das publicações científicas da Fisioterapia no campo da SC no Brasil.

\section{METODOLOGIA}

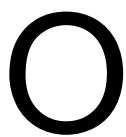
presente estudo consiste em uma revisão de literatura, a partir do qual foram realizadas buscas nas bases de dados Bireme, SciELO e Lilacs com os descritores "Fisioterapia" e "Saúde Coletiva" entre junho de 2015 e fevereiro de 2016, com o descritor boleano "and". A escolha de apenas dois descritores justifica-se por uma opção metodológica que teve por objetivo analisar a identidade das publicações que relacionam a Fisioterapia com a SC a fim de entender como se dá a identificação da profissão com esse campo da saúde. 
Os critérios de inclusão foram artigos nacionais que relacionassem a Fisioterapia à SC e que estivessem disponíveis na integra. Excluíram-se os estudos que não discutiam pelo menos um dos pressupostos do campo: conceito ampliado de saúde, ações de saúde como prática social, a determinação social das doenças e atuação interdisciplinar ${ }^{4}$. A inclusão apenas de artigos nacionais justifica-se pela escolha de estudar a articulação da fisioterapia no contexto da Saúde Coletiva brasileira, com suas especificidades ${ }^{5}$.

$\mathrm{Na}$ primeira busca estratégica com os descritores "Fisioterapia" e "Saúde Coletiva", encontraram-se 108 publicações, porém apenas 45 estavam disponíveis na integra. Dessas 45 publicações, 27 foram excluídas: 10 eram estudos com experiências internacionais, 16 não tratavam do tema abordado neste estudo e 5 eram artigos repetidos, restando 14 artigos selecionados para esta revisão (Figura 1). Não foi delimitado o período de publicação durante a busca, ou seja, todas publicações foram incluídas independentemente do período de publicação.

FIGURA 1. Fluxo de seleção de artigos na revisão narrativa sobre Fisioterapia e Saúde Coletiva: análise crítica da produção científica no Brasil

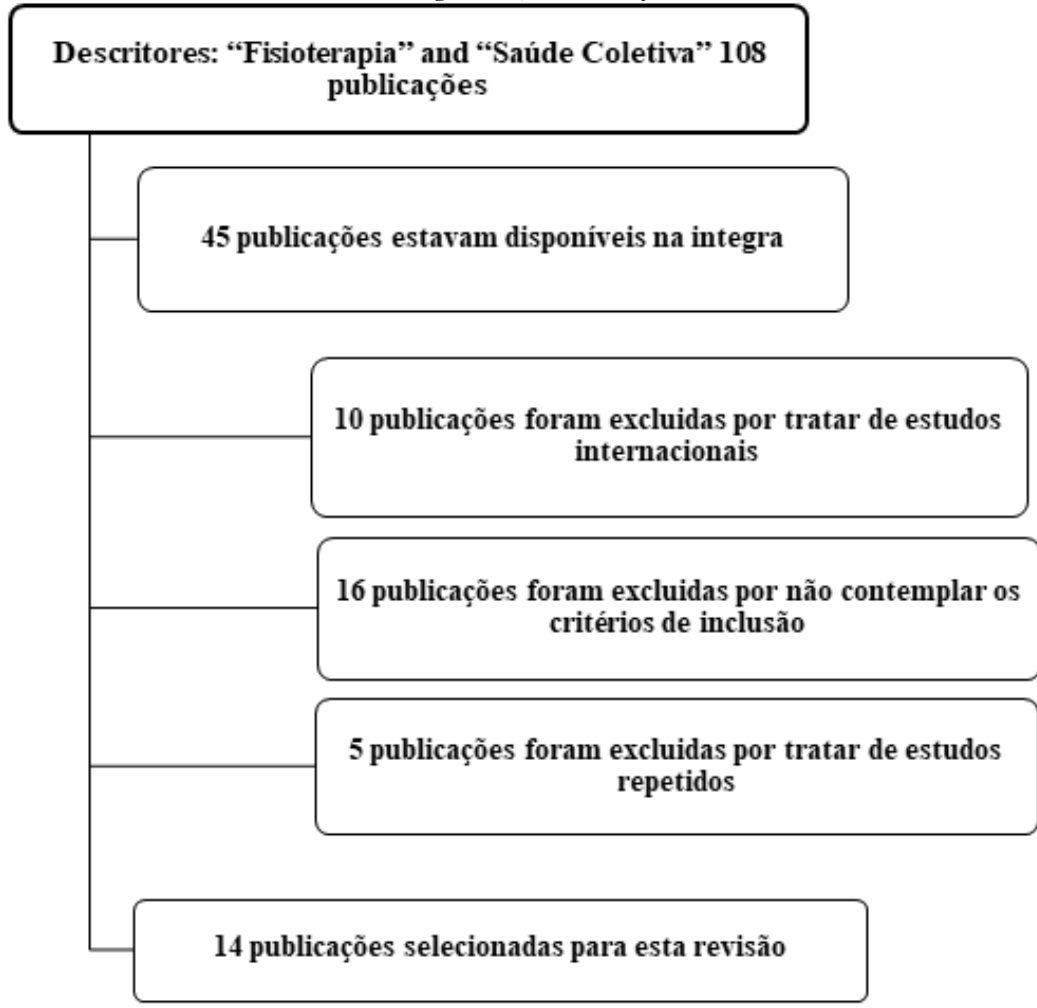

Os artigos foram caracterizados quanto ao nível de atenção à saúde, revista e região de publicação e analisados quanto ao conteúdo, sendo definidos três eixos centrais para análise crítica do conteúdo: 1) Formação para Saúde Coletiva ou Saúde Pública; 2) Integralidade nas práticas da Fisioterapia e Saúde Coletiva; 3) Interdisciplinaridade nas práticas da Fisioterapia e Saúde Coletiva. A escolha dos eixos foi definida por meio da leitura dos artigos e identificação do tema central em cada estudo. Os artigos foram divididos em alguns conteúdos de forma mutuamente excludente, em que cada artigo selecionado foi discutido em apenas um tópico da discussão. É importante ressaltar que a inclusão dos artigos em suas respectivas categorias foi realizada considerando a ênfase no tema correspondente, e não sua abordagem exclusiva naquele tema. 


\section{RESULTADOS E DISCUSSÃO}

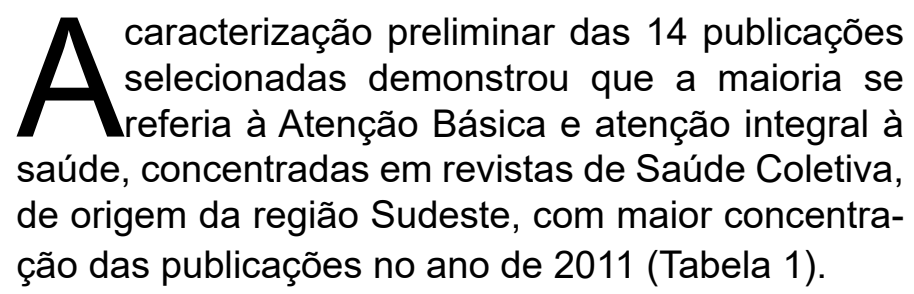

TABELA 1. Apresentação dos artigos selecionados por ano e revista de publicação, autor, título e nível de atenção

\begin{tabular}{|c|c|c|c|}
\hline Revista/Local/Ano & Autores & Título & Nível de atenção \\
\hline IFF/Fiocruz 2005 & Ungier R. & $\begin{array}{c}\text { Interações biomecânicas entre a organização postural global e a } \\
\text { respiração: um olhar ampliado sobre a fisioterapia dirigida a crianças } \\
\text { com doença respiratória }\end{array}$ & $\begin{array}{c}\text { Média } \\
\text { complexidade }\end{array}$ \\
\hline Rev. APS 2007 & Custódio et al. & $\begin{array}{l}\text { Contribuições da fisioterapia para a promoção de saúde do } \\
\text { cuidador informal }\end{array}$ & Atenção Básica \\
\hline $\begin{array}{l}\text { Revista Saúde e } \\
\text { Sociedade } 2008\end{array}$ & $\begin{array}{l}\text { Salmória JG, } \\
\text { Camargo WA. }\end{array}$ & $\begin{array}{l}\text { Uma Aproximação dos Signos - Fisioterapia e Saúde - } \\
\text { aos Aspectos Humanos e Sociais }\end{array}$ & Atenção Integral \\
\hline $\begin{array}{l}\text { Revista Fisioterapia e } \\
\text { Movimento } 2010\end{array}$ & Barbosa et al. & $\begin{array}{l}\text { Experiência da fisioterapia no Núcleo de Apoio à Saúde da } \\
\text { Família em Governador Valadares, MG }\end{array}$ & Atenção Básica \\
\hline $\begin{array}{l}\text { Revista Ciência \& Saúde } \\
\text { Coletiva } 2010\end{array}$ & Bispo-Júnior JP. & $\begin{array}{c}\text { Fisioterapia e saúde coletiva: desafios e novas responsabilidades } \\
\text { profissionais }\end{array}$ & Atenção Integral \\
\hline $\begin{array}{l}\text { Escola Nacional de } \\
\text { Saúde Pública Sergio } \\
\text { Arouca, } 2009\end{array}$ & Araújo JCS. & $\begin{array}{c}\text { Inserção da fisioterapia na atenção básica: um caminho necessá- } \\
\text { rio a percorrer em saúde pública }\end{array}$ & Atenção Básica \\
\hline $\begin{array}{l}\text { Revista Ciência e Saúde } \\
\text { Coletiva } 2011\end{array}$ & Naves CR, Brick VS. & $\begin{array}{l}\text { Análise quantitativa e qualitativa do nível de conhecimento dos } \\
\text { alunos do curso de fisioterapia sobre a atuação do fisioterapeuta } \\
\text { em saúde pública }\end{array}$ & Atenção Básica \\
\hline $\begin{array}{l}\text { Revista História, } \\
\text { Ciências, Saúde - } \\
\text { Manguinhos } 2011\end{array}$ & Faria L, Santos LAC. & As profissões de saúde: uma análise crítica do cuidar & Atenção Integral \\
\hline $\begin{array}{l}\text { Revista Interface - } \\
\text { Comunicação, Saúde, } \\
\text { Educação } 2011\end{array}$ & $\begin{array}{l}\text { Neves LMT, Aciole } \\
\text { GG }\end{array}$ & $\begin{array}{l}\text { Desafios da integralidade: revisitando as concepções sobre o } \\
\text { papel do fisioterapeuta na equipe de Saúde da Família }\end{array}$ & Atenção Básica \\
\hline
\end{tabular}

Revista Trabalho Gonçalves FG, Carva- O ensino da Saúde Coletiva na Universidade Estadual de Londrina: da Atenção Integral Educação e Saúde 2012 lho BG, Trelha CS análise documental à percepção dos estudantes

\begin{tabular}{cccc}
$\begin{array}{c}\text { Revista brasileira pro- } \\
\text { moção e saúde 2012 }\end{array}$ & Rodrigues et al. & $\begin{array}{c}\text { Conhecimento e interesse em saúde pública: opiniões dos alu- } \\
\text { nos de graduação em fisioterapia }\end{array}$ & Atenção Integral \\
\hline $\begin{array}{c}\text { Revista Fisioterapia e } \\
\text { Pesquisa 2013 }\end{array}$ & $\begin{array}{c}\text { Seriano KN, Muniz } \\
\text { VRC, Carvalho MEIM. }\end{array}$ & $\begin{array}{c}\text { Percepção de estudantes do curso de fisioterapia sobre sua } \\
\text { formação profissional para atuação na atenção básica } \\
\text { no Sistema Único de Saúde }\end{array}$ & Atenção Básica \\
\hline $\begin{array}{c}\text { Revista baiana de } \\
\text { saúde pública 2013 }\end{array}$ & $\begin{array}{c}\text { Medeiros DKS, } \\
\text { Neves RF. }\end{array}$ & $\begin{array}{c}\text { Análise crítica das práticas na atenção primária à saúde com base nos } \\
\text { relatos dos estudantes do curso de fisioterapia }\end{array}$ & Atenção Básica \\
\hline
\end{tabular}

Revista Fisioterapia e Almeida SM, Martins Integralidade e formação para o Sistema Único de Saúde na Pesquisa $2014 \quad$ AM, Escalda PMF. perspectiva de graduandos em Fisioterapia

Atenção Integral 


\section{a) Formação para Saúde Coletiva ou Saúde Pública}

Os Descritores em Ciências da Saúde (DeCS) da Biblioteca Virtual em Saúde (BVS) apresentam como resultado de busca o termo SC como sinônimo de Saúde Pública (SP). Essa semelhança semântica pode ser encontrada na maioria dos estudos levantados por esta revisão. A SC pode ser definida como um campo de produção de conhecimentos voltado para a compreensão da saúde e para a explicação de seus determinantes sociais, bem como o âmbito de práticas direcionadas prioritariamente para sua promoção, além de voltadas para a prevenção e o cuidado a agravos e doenças, tomando por objeto não apenas os indivíduos, mas sobretudo os grupos sociais ${ }^{6,7}$.

Os autores da SC apresentam este campo como uma ampliação da atuação da SP, necessária para uma melhor compreensão da situação de saúde dos indivíduos e coletividades, para além da proposição de práticas intervencionistas do Estado, ou representantes desse nos serviços de saúde. Assim, a SC avançaria na contribuição da compreensão da saúde em seu conceito ampliado, não se limitando a um campo focado na ocorrência da doença. A intervenção em doenças é marcante nas discussões da SP, tal fato pode ser observado por Winslow ${ }^{8}$ na origem do seu conceito:

A Saúde Pública é a ciência e a arte de prevenir a doença, prolongar a vida, promover a saúde física e a eficiência através dos esforços da comunidade organizada para o saneamento do meio ambiente, o controle das infecções co-

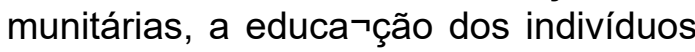
nos princípios de higiene pessoal [...].

Apesar das publicações selecionadas na presente revisão não avançarem na discussão das diferenças de conceitos, todas parecem discutir a SP ou SC como campo de práticas, e, a maioria, como práticas na Atenção Básica à Saúde (ABS). Talvez este fato esteja relacionado com a recente instituição da presença do fisioterapeuta como profissional do Núcleo de Apoio à Saúde da Família (NASF) ${ }^{9}$, o que influenciou diretamente a inclusão de conteúdos de ABS nas grades curriculares das graduações de fisioterapia.

Além da confusão semântica da SC com a SP, a maioria dos estudos selecionados nesta revisão relaciona a atuação no SUS a uma articulação da Fisioterapia com a SC, porém a prestação da assistência em um serviço público de saúde não necessariamente significa alguma aproximação com saberes e práticas da SC. Afinal, o profissional de saúde pode ter inserção no SUS e não necessariamente seguir os pressupostos da SC, ou mesmo exercer suas práticas em um serviço privado de saúde baseado nos conceitos da SC. A aproximação da Fisioterapia com o campo da SC não deveria se expressar apenas por meio da atenção assistencial em saúde, podendo extrapolar e transitar por outros áreas da atenção à saúde. A SC apresenta disciplinas básicas, como: a epidemiologia, o planejamento e administração de saúde e as ciências sociais em saúde, fundamentando um âmbito de práticas transdisciplinar, multiprofissional, interinstitucional e transetorial ${ }^{10}$.

Os estudos estabelecem uma relação direta dos termos SP e/ou SC com a ABS, restringindo a atuação a este nível de atenção e considerando que essa inserção do fisioterapeuta representa uma das perspectivas da aproximação da Fisioterapia e SC. Esse fato é pontuado por Gonçalves, Carvalho e Trelha ${ }^{11}$ por intermédio da análise do discurso dos estudantes que utilizam os termos como sinônimos e poucos conseguem relacionar a SC à atenção ambulatorial e/ou hospitalar ${ }^{11}$. A atuação como trabalhador de saúde na ABS não necessariamente indica uma atuação sobre os pressupostos da SC, podendo representar uma reprodução da perspectiva do modelo hospitalocêntrico/ curativista na oferta de acesso aos serviços de saúde, direcionando a atenção prioritariamente às doenças.

Gonçalves, Carvalho e Trelha ${ }^{11}$ também identificam que as atividades em grupos realizadas na Unidade de Saúde da Família (USF) são dirigidas geralmente a indivíduos com uma doença instalada, como grupos de pessoas com hipertensão, diabetes e lombalgias ${ }^{11}$. Os estudos de Naves e Brick ${ }^{12}$ e Bispo Júnior $^{3}$ ampliam a perspectiva da fisioterapia na SC por meio da atuação na participação em Programas Institucionais, Saúde do Trabalhador e Vigilância Sanitária, para além das ações básicas de saúde, o que difere dos estudos de Rodrigues et al. ${ }^{13}$ e Seriano, Muniz e Carvalho ${ }^{14}$, em que a SC se expressa apenas por meio do relato da atuação do fisioterapeuta da ABS, voltados para atuação preventiva.

Nos estudos de Naves e Brick ${ }^{12}$ e Rodrigues et al. ${ }^{13}$, os estudantes consideram o próprio conhecimento relacionado com a SP limitado. Esse resultado diverge do estudo de Seriano, Muniz e Carvalho ${ }^{14}$, no qual os estudantes referem ter recebido informações teóricas suficientes sobre o SUS. Sobre os estágios curriculares obrigatórios voltados para ABS, os estudos de Rodrigues et al. ${ }^{13}$ e Seriano, Muniz e CarvaIho ${ }^{14}$ identificam que estes estão concentrados geralmente no último semestre, enquanto os estágios hospitalares possuem carga horária mais elevada e se iniciam nos semestres anteriores, influenciando diretamente na preparação e interesse de atuação dos acadêmicos. 
Os alunos dos semestres iniciais referem pouco interesse no estudo da SP, enquanto os acadêmicos do último semestre relatam ter bastante interesse, referindo uma preocupação com o mercado de trabalho ${ }^{13}$. A maioria dos estudantes entrevistados expressa a possibilidade de atuação tanto no setor público quanto no privado em suas vidas profissionais14. Esse resultado poderia ser justificado por essa preocupação com a inserção no mercado de trabalho, e não necessariamente com o interesse de atuar no SUS por identificação com o projeto de saúde da Reforma Sanitária.

A formação voltada para a SP visa a um foco para uma atuação no sistema de saúde vigente, investindo na integralidade da atenção à saúde, em conformidade com os princípios e as diretrizes do SUS ${ }^{15}$ garantindo a formação preconizada pelas DCN nos diversos níveis de atenção ${ }^{16}$, sem compreender a SP como mais uma área de especialização e qualificação do fisioterapeuta ${ }^{12}$. Salmoria e Camargo $^{17}$ e Gonçalves, Carvalho e Trelha ${ }^{11}$ destacam que a articulação da Fisioterapia com as ciências sociais permite um olhar histórico-humano-social sobre as ações de saúde e da prevenção de doenças, podendo ser abordada conjuntamente com outros conteúdos específicos da profissão ${ }^{11,17}$, garantindo a formação generalista preconizada pelas $\mathrm{DCN}$ nos diversos níveis da atenção ${ }^{16}$.

Independentemente das escolhas no uso das terminologias de SC ou SP, reconhecidas as semeIhanças ou diferenças, ambas perspectivas colaboram para a elaboração das DCN do curso de Fisioterapia. Ambas indicam que o egresso/profissional deve ter uma formação generalista, humanista, crítica e reflexiva, com capacidade de atuar em todos os níveis de atenção e em equipe multiprofissional, inter e transdisciplinar, integrando-se em programas de promoção, prevenção, proteção, reconhecendo a saúde como direito, e garantindo a integralidade da assistência $^{16}$. Essas competências se aproximam dos valores pressupostos no campo da SC e do sistema de saúde vigente no país, o SUS.

\section{b) Integralidade nas práticas da Fisioterapia e Saúde Coletiva}

A integralidade é considerada como um dos conceitos mais caros às novas propostas em SC, e este princípio se aproxima da valorização das subjetividades e singularidades de cada paciente e se distancia do paradigma biomédico mecanicista e reducionista ${ }^{18}$. Almeida, Martins e Escalda ${ }^{19}$ concordam com essa percepção quando detectam por meio da percepção dos estudantes a integralidade como atributo da prática do fisioterapeuta, indicando que a abordagem deve ultrapassar o reducionismo do cuidado biomédico.

Os resultados obtidos por Ungier ${ }^{18}$ apontam para a relevância da construção de um modelo ampliado de fisioterapia, que vise à problematização do papel do profissional no âmbito da SC e lance luz sobre sua responsabilidade, não apenas quanto ao objeto de estudo dela, que neste caso é a melhora do quadro pulmonar no tratamento de crianças com doença respiratória, mas no que diz respeito à promoção da autonomia e da qualidade de vida do paciente. Ungier ${ }^{18}$ baseia seu trabalho em conceitos que dialogam com uma proposta integrada de saúde, descartando o aspecto fragmentário do modelo biomédico tradicional e dando valor a totalidade do indivíduo no que diz respeito a não divisão do corpo em partes isoladas e finaliza seu trabalho afirmando que a "nova Saúde Pública" carece de uma concepção de "nova fisioterapia", em que a parte não esteja fora do todo, em que corpo, vivências e história sejam contemplados de forma integral, e que cada paciente seja único.

Medeiros e Neves $^{20}$ identificam, por meio do discurso de acadêmicos a respeito do estágio em SC, uma compreensão da integralidade como resposta à complexidade, em que os casos de mais fácil resolução não recebem uma abordagem ampliada. Bem como a integralidade sendo usada como jargão, remetendo ao conceito ampliado de saúde preconizado pela Organização Mundial de Saúde (OMS), que muitas vezes engessa a prática profissional devido à perspectiva utópica de saúde. Os estudantes de fisioterapia compreendem a importância da integralidade, mas nem sempre conseguem conciliá-la com a prática, identificando também que apenas a conduta da fisioterapia é insuficiente ante as demandas que surgem como: limitações na estrutura domiciliar de atendimento, barreiras geográficas, demandas de ordem socioeconômica e das relações de cuidado envolvendo a família, além do insuficiente suporte técnico assistencial de trabalhadores em saúde no território ${ }^{20}$.

Essa constatação aponta para a necessidade de os currículos acadêmicos promoverem uma reformulação, como citado por Almeida, Martins e Escal$\mathrm{da}^{19}$, quando os autores pontuam limitações devido à desarticulação da teoria e prática, resultando em tentativas isoladas de abordagem integral na formação, e afirmam existir uma dificuldade de incorporação da integralidade como uma temática transversal a todas disciplinas em diferentes áreas do conhecimento, assim como nas atividades práticas, estágios e projetos de pesquisa, extensão ${ }^{19}$.

Araújo ${ }^{21}$ discute em seu trabalho a importância e a qualificação do atendimento em saúde, sob a ótica da integralidade, considerando esse princípio 
um dos norteadores para a inclusão do profissional na $A B S^{21}$, além de ser uma diretriz do SUS, entendida como conjunto articulado e contínuo das ações e serviços preventivos e curativos, individuais e coletivos, exigidos para cada caso em todos os níveis de complexidade do sistema de saúde ${ }^{22}$.

Existe um consenso, nos estudos revisados, quanto à importância da incorporação da integralidade na prática fisioterapêutica para realização de um trabaIho mais eficaz, assim como um consenso quanto ao distanciamento desse princípio da prática profissional decorrente de uma formação focada em especialidades, que não promove a interdisciplinaridade e fragmenta o ensino, resultando na falta de capacitação de profissionais capazes de desenvolver um olhar crítico, humano e integral sobre o processo do cuidado.

Medeiros e Neves ${ }^{20}$ problematizam a organização dos conteúdos curriculares de fisioterapia ao afirmar que o discurso escrito no projeto político muitas vezes se apresenta distante de uma prática baseada nas demandas sociais e nas políticas públicas de saúde. As autoras apontam que a valorização apenas do conhecimento biológico é um grande desafio à integralidade, pois o profissional não se torna apto a identificar os determinantes sociais em saúde, deixando de contribuir para o processo de autonomia e participação social dos usuários. Segundo as autoras, a universidade é um espaço de contradição que consolida e perpetua as desigualdades sociais, mas apontando que nela também se encontra um movimento contra a ordem que repensa a produção desses conhecimentos gerados e tenta aproximá-los das classes populares ${ }^{20}$.

Faz-se necessário, portanto, disputar os espaços da universidade, seja no ensino, pesquisa e extensão, para contribuir para o processo de reformulação dos conteúdos curriculares em saúde e dos conhecimentos ali produzidos, aproximando-os das necessidades reais da população, dos princípios e diretrizes do SUS e contribuindo para formação de novos profissionais em saúde que exerçam e lutem por práticas mais integrais e articuladas com os pressupostos da SC.

\section{c) Interdisciplinaridade nas práticas da Fisioterapia e Saúde Coletiva}

As publicações que referem práticas interdisciplinares localizam suas práticas na $A B S$, isso porque esse parece ser lócus privilegiado pelas características das suas ferramentas de gestão do trabalho, como a equipe de referência e apoio matricial ${ }^{23}$.

A interdisciplinaridade se apresenta como um grande desafio para prática em saúde e pontua que a participação dos estudantes nas ações básicas de saúde contribui para a formação de um profissional generalista capaz de compreender a relação da integralidade em saúde e realizar uma prática direcionada para as demandas da comunidade. Apesar desse reconhecimento, as possibilidades de práticas interdisciplinares são raras durante a formação nos cursos de graduação em saúde, uma vez que esses possuem currículos fechados e com grande número de disciplinas obrigatórias, impossibilitando que os estudantes compartilhem matérias teóricas comuns e práticas integradas ${ }^{24}$.

O processo de trabalho do fisioterapeuta na ABS deve suprir a demanda da comunidade, reduzindo danos e agravos, com uma prática integral que perpasse o acolhimento, atendimento individual na unidade, atendimento domiciliar, grupos operativos e atividades educativas em equipe. Alguns problemas são apontados como dificuldades, como a falta integração do NASF com as equipes das ESF, a formação clínica e práticas isoladas comprometendo a atenção integral à comunidade. Essa desarticulação do NASF com as equipes da ESF reforça a importância do trabalho interdisciplinar na ABS e a importância de experiências interdisciplinares acontecerem desde o processo de formação dos profissionais de saúde. O maior desafio para as práticas de SC é uma formação profissional que não seja baseada apenas em especialidades, para que a profissão se adapte às propostas do SUS e consiga realizar um trabalho de fato interdisciplinar ${ }^{25}$. 
Neves e Aciole ${ }^{26}$ realizam uma revisão de literatura para entender a demanda de atuação do fisioterapeuta na SC, com ênfase na ESF, analisando a definição do papel e campo de trabalho do fisioterapeuta. Uma das dificuldades referidas se deve ao fato de suas atribuições serem desconhecidas pelos gestores e profissionais da equipe, como também a visão interdisciplinar da saúde estar em processo de construção nas atividades dos profissionais. Outra dificuldade encontrada seria a indeterminação do papel do fisioterapeuta dentro de uma abordagem multidisciplinar e interdisciplinar, ou seja, uma falta de clareza sobre o objeto de trabalho da fisioterapia ${ }^{26}$.

Essa indefinição em relação à atuação do fisioterapeuta também se deve a outros fatores, como: desarticulação da formação acadêmica e prática profissional, afastamento da categoria aos conceitos da SP e da SC e preservação do paradigma reabilitador do fisioterapeuta. Outra questão apresentada é o reconhecimento do fisioterapeuta na ESF apenas em sua função reabilitadora, que dificulta uma atuação profissional que extrapole outros horizontes de intervenção, por exemplo, na promoção e prevenção, devido também a uma demanda assistencial reprimida pelo sistema de saúde.

Faria e Santos ${ }^{27}$ realizam uma análise crítica do cuidar sobre a perspectiva da atuação da fisioterapia e da enfermagem, com destaque ao desafio do trabalho multiprofissional e interdisciplinar na formação de novos profissionais de saúde, assim como em suas práticas, buscando a humanização do cuidado de forma integrada. Segundo as autoras, o cuidado não pode ser visto como uma menor atividade nem ser visto apenas como o cuidado da doença, e, sim, do sujeito que ali se apresenta composto de diversas complexidades e determinantes. O estudo ressalta que a aplicação de técnicas não é suficiente se o fisioterapeuta não atuar em articulação com os demais profissionais de saúde, em um trabalho interdisciplinar. As autoras referem a oportunidade para que os fisioterapeutas reflitam sobre o desafio do redirecionamento de suas práticas, compreendendo sua dimensão social, exercitando o trabalho interdisciplinar com a equipe de saúde e capacitando-se como um profissional de saúde de fato, não apenas um profissional da reabilitação que atua de forma segmentada ${ }^{27}$.

\section{CONCLUSÃO}

\footnotetext{
$\mathrm{O}$ bservou-se, com o presente estudo, que a produção científica da Fisioterapia na Saúde Coletiva cresceu nos últimos dez anos, apresentando maior concentração no ano de 2011, provavelmente devido à regulamentação da profissão na Atenção Básica, mas ainda representando uma produção incipiente no que se refere às demais produções científicas na Fisioterapia. A maior parte dos estudos explora as práticas assistenciais, sem extrapolar para uma reflexão crítica sobre os pressupostos e características da atuação no campo da Saúde Coletiva que perpasse a assistência em saúde.

Pode-se perceber que existe uma dificuldade do profissional fisioterapeuta em realizar práticas integrais e interdisciplinares dentro dos serviços devido a uma desarticulação da formação profissional com a prática em saúde. Isso reafirma o grande desafio de aproximar o profissional fisioterapeuta do campo da SC, para que isso reverbere não somente na sua prática assistencial, mas também para que esses profissionais estejam capacitados para contribuir para a construção de política públicas, para o levantamento de dados epidemiológicos de saúde, para a gestão de serviços de saúde e para a articulação da saúde com as ciências sociais, enfim, com uma contribuição para a atenção integral à saúde.
} 


\section{Referências}

1. Bispo-Júnior JP. Fisioterapia e Saúde Coletiva - reflexões, fundamentos e desafios. 1 ed. São Paulo: Hucitec; 2013.

2. Coffito. Conselho Federal de Fisioterapia e Terapia Ocupacional. Resolução n. ${ }^{\circ} 363$, de 20 de maio de 2009. Reconhece a Fisioterapia em Saúde Coletiva como especialidade do profissional Fisioterapeuta e dá outras providencias. Diário Oficial da União, Brasília, DF, 16 jun. 2009.

3. Bispo-Júnior JP. Fisioterapia e saúde coletiva: desafios e novas responsabilidades profissionais. Ciênc. saúde coletiva. 2010. 15(1): 1627-1636.

4. Paim JS, Almeida-filho N. Saúde coletiva: uma "nova saúde pública" ou campo aberto a novos paradigmas? Rev. Saúde Pública. 1998. 32(4): 299-316.

5. Vieira-da-Silva LM, Paim JS, Schraiber LB. O que é Saúde Coletiva. In: Paim, JS, Almeida-filho N (Org.). Saúde coletiva: Teoria e prática. Rio de Janeiro: MedBook; 2013. p. 3.

6. Paim JS. Desenvolvimento teórico-conceitual do ensino em saúde coletiva. In: ABRASCO. Rev. Ensino da Saúde pública, Medicina Preventiva e Social no Brasil. 1982; 1:3-19.

7. Donnangelo MCF. A pesquisa em Saúde Coletiva no Brasil - A Década de 70. In: ABRASCO. Rev. Ensino da Saúde Pública, Medicina Preventiva e Social no Brasil. 1983; 2:17-35.

8. Winslow CEA. The Untilled fields of public health. Sciense. 1920; 51: p.23.

9. Brasil. Ministério da Saúde. Portaria GM/MS No 154, de 24 de janeiro de 2008. Cria os Núcleos de Apoio à Saúde da Família. Diário Oficial da União, Brasília, DF, 2008.

10. Paim JS, Almeida-Filho N. A Crise da Saúde Pública e a Utopia da Saúde Coletiva. Salvador: Casa da Qualidade Editora; 2000.

11. Gonçalves FG, Carvalho BG, Trelha CS. O ensino da Saúde Coletiva na Universidade estadual de Londrina: da análise documental à percepção dos estudantes. Trab. educ. saúde. 2012; 10(2): 301-314.

12. Naves CR, Brick VS. Análise quantitativa e qualitativa do nível de conhecimento dos alunos do curso de fisioterapia sobre a atuação do fisioterapeuta em saúde pública. Ciênc. Saúde coletiva. 2011; 16(1): 1525-1534.

13. Rodrigues JE, Gomes CAFP, Filho AVD, Nascimento MV, De Souza JCC, Pontes-Barros JF. Conhecimento e interesse em saúde pública: opiniões dos alunos de graduação em fisioterapia. Rev Bras Promoç Saúde. 2012; 25(1): 59-64.

14. Seriano KN, Muniz VRC, Carvalho MEIM. Percepção de estudantes do curso de fisioterapia sobre sua formação profissional para atuação na atenção básica no Sistema Único de Saúde. Fisioter. Pesqui. 2013; 20(3): 250-255.

15. Batista KBC, Gonçalves OSJ. Formação dos Profissionais de Saúde para o SUS: significado e cuidado. Saúde Soc. 2011; 20(4): 884-899.

16. Brasil. Ministério da Educação. Resolução CNE/CES 4, de 19 de fevereiro de 2002. Institui diretrizes curricula res nacionais do curso de graduação em fisioterapia. Diário Oficial da União, Brasília, DF, 04 de março 2002.

17. Salmória JG, Camargo WA. Uma Aproximação dos Signos - Fisioterapia e Saúde - aos Aspectos Humanos e Sociais. Saúde Soc. 2008; 17(1): 73-84.

18. Ungier R. Interações biomecânicas entre a organização postural global e a respiração: um olhar ampliado sobre a fisioterapia dirigida a crianças com doença respiratória [dissertação]. Rio de Janeiro: IFF/FIOCRUZ; 2005.

19. Almeida SM, Martins AM, Escalda PMF. Integralidade e formação para o Sistema Único de Saúde na perspectiva de graduandos em Fisioterapia. Fisioter. Pesqui. 2014; 21(3): 271-278.

20. Medeiros DKS, Neves RF. Análise crítica das práticas na atenção primária à saúde com base nos relatos dos estudantes do curso de fisioterapia. Rev. Baiana de Saúde Pública. 2013; 37(1):87-105.

21. Araújo JCS. Inserção da fisioterapia na atenção básica: um caminho necessário a percorrer em saúde pública [dissertação]. Porto Alegre (RS): Escola Nacional de Saúde Pública Sérgio Arouca; 2009.

22. Brasil. Lei $n^{\circ} 8.080$, de 19 de setembro de 1990. Dispõe sobre as condições para a promoção, proteção e recuperação da saúde, a organização e o funcionamento dos serviços correspondentes e dá outras providências. Diário Oficial da República Federativa do Brasil, Poder Executivo, Brasília, DF, 20 set. 1990.

23. Campos GWS, Domitti AC. Apoio matricial e equipe de referência: uma metodologia para gestão do trabalho interdisciplinar em saúde. Cad. Saúde Pública. 2007; 23(2): 399-407.

24. Custódio LC, Oliveira BWA, Machado Neto CD, Moraes FAP, Moura RAA. Contribuições da fisioterapia para a promoção de saúde do cuidador informal. Rev. APS. 2007; 10(1): 81-83.

25.Barbosa EG, Ferreira DLS, Furbino SAR, Ribeiro EEN. Experiência da Fisioterapia no Núcleo de Apoio à Saúde da Família em Governador Valadares, MG. Fisioter. Mov. 2010; 23(2): 323-330.

26. Neves LMT, Aciole GG. Desafios da integralidade: revisitando as concepções sobre o papel do

fisioterapeuta na equipe de Saúde da Família. 2011; 15(37): 551-64.

27. Faria L, Santos LAC. As profissões de saúde: uma análise crítica do cuidar. Hist. Cienc.

Saúde-Manguinhos. 2011; 18(1): 227-240. 